\title{
Rehabilitating a brain with Alzheimer's: a proposal
}

This article was published in the following Dove Press journal:

Clinical Interventions in Aging

23 February 201I

Number of times this article has been viewed

\author{
Gonzalo Emiliano \\ Aranda-Abreu ${ }^{1,3}$ \\ María Elena \\ Hernández-Aguilar ${ }^{1,3}$ \\ Jorge Manzo Denes ${ }^{1,4}$ \\ Luis Isauro García \\ Hernández ${ }^{1,4}$ \\ Marisol Herrera Rivero \\ 'Programa de Neurobiología, \\ ${ }^{2}$ Doctorado en Ciencias Biomédicas, \\ Universidad Veracruzana, Xalapa, \\ Veracruz, México, ${ }^{3}$ Cuerpo Académico \\ de Neuroquímica, ${ }^{4}$ Cuerpo Académico \\ de Neurociencias.
}

\begin{abstract}
Alzheimer's disease (AD) is the most common neurodegenerative disorder, originating sporadically in the population aged over 65 years, and advanced age is the principal risk factor leading to AD development. In spite of the large amount of research going on around the globe and all the information now available about $\mathrm{AD}$, there is still no origin or triggering process known so far. Drugs approved for the treatment of AD include tacrine, donepezil, rivastigmine, galantamine, and memantine. These may delay or slow down the degenerative process for a while, but they can neither stop nor reverse its progression. Because that this might be due to a lack of effect of these drugs on degenerating neurons, even when they are able to potentiate the brain in nondegenerative conditions, we propose here an alternative therapy consisting of initial repair of neuronal membranes followed by conventional drug therapies. The rehabilitation of neurons in a degeneration process would enable the drugs to act more effectively on them and improve the effects of treatment in AD patients.
\end{abstract}

Keywords: Alzheimer, rehabilitation, drugs

\section{Introduction}

Alzheimer's disease (AD) is a neurodegenerative disorder with loss of memory and other cognitive functions which progresses slowly, extending the disease for several years. Survival is very variable in these patients, although death often occurs within 10 years of onset, often as a result of infections.

In more than $90 \%$ of cases, $\mathrm{AD}$ develops after the age of 65 years, and doubles its prevalence with every successive decade of life, from $10 \%$ at $60-70$ years to $40 \%$ at 80 years of age. Several chromosomes have been shown to be implicated in the pathology of $\mathrm{AD}$, including chromosomes 1, 14, and 21, associated with the familial early-onset forms of the disease, and chromosomes 12 and 19, linked to late-onset forms. ${ }^{1}$ However, most cases cannot be explained genetically, and thus several hypotheses have been raised over the years in an attempt to explain this complex disease, some of them suggesting the presence of unidentified infectious or toxic agents $^{2}$ which modify the risk of developing AD.

One of the main processes leading to the symptomatology of $\mathrm{AD}$ is the abnormal phosphorylation of tau, a microtubule-associated protein. When tau gets hyperphosphorylated, it dissociates from microtubules, and structures called paired helical filaments begin to form within the cell bodies of neurons, generating neurofibrillary tangles, with hyperphosphorylated tau as their main component and leaving a loose cytoskeleton, which leads the neuronal membrane to lose its shape. 
According to the amyloid cascade hypothesis, ${ }^{3} \beta$-amyloid peptide, the principal constituent of neuritic plaques, may have a major role in the neuropathology of $\mathrm{AD}$. $\beta$-amyloid peptide generally exists in two forms, one is 40 amino acids long and the other is 42 amino acids long $\left(A \beta_{40}\right.$ and $A \beta_{42}$, respectively), which differ in their terminal carbon structure. Of these two forms, $\mathrm{A} \beta_{42}$ is the most prone to aggregation. ${ }^{4}$ Amyloid peptides are generated by the sequential actions of two enzymes, ie, $\beta$-secretase (BACE1) and $\gamma$-secretase.

BACE1 is widely expressed in the brain (mainly in the hypothalamus), and its involvement in the myelination process of the peripheral nervous system has been established. ${ }^{5}$ Even though the function of BACE1 is not fully understood as yet, recent studies demonstrate that it activates neuroregulin-1, thus having direct involvement in the myelination process. ${ }^{6} \gamma$-secretase is a proteic presenilin complex, and one of its largest components is a protein known as nicastrin, the function of which has been widely associated with embryonic growth retardation and mortality.

There is no cure for $\mathrm{AD}$ as yet, and drug therapies are not effective enough to avoid symptoms. For these reasons, we felt the need to come up with an alternative, accessible, and safe therapy able to augment conventional drug treatments and have increased effectiveness in $\mathrm{AD}$ patients at any stage of the disease, but mainly in the first stages, where the rehabilitation of degenerating neurons could make a great difference to progression of the disease.

\section{Drugs used for AD treatment}

Five drugs have been approved for AD treatment in different stages of the disease, although they all show limited efficacy. These are tacrine, donepezil, rivastigmine, galantamine, and memantine. ${ }^{8}$ Tacrine was one of the first drugs to be used for memory loss and cognitive decline, often accompanied by abnormal behavior and physical debilitation in AD patients. The alleged success of tacrine in treating these symptoms was heralded as confirmation of the cholinergic theory of AD. Nevertheless, its efficacy remains controversial. ${ }^{9}$ A acetylcholinesterase inhibitor, tacrine has been associated with increased levels of transaminases in approximately 50\% of the patients treated, but the mechanism by which it causes damage is not completely understood, and there could be genetic factors involved. ${ }^{10}$

Donepezil is another acetylcholinesterase inhibitor its selectiveness as an inhibitor is fairly specific. Donepezil is used for the treatment of moderate to severe $\mathrm{AD},{ }^{11}$ and is apparently well tolerated by patients and has few side effects. ${ }^{12}$ On the other hand, rivastigmine has shown fewer side effects and better acceptance by patients ${ }^{13}$ than donepezil. However, the effectiveness of donepezil has been limited in patients with $\mathrm{AD} .{ }^{14}$

Galantamine, another member of the acetylcholinesterase inhibitor group, has shown a protective role for cortical neurons, preventing them from the cytotoxicity generated by aggregation of amyloid peptides. ${ }^{15,16}$

Unlike most drugs, memantine is not an acetylcholinesterase inhibitor, but an $\mathrm{N}$-methyl-D-aspartate receptor antagonist. It reduces clinical deterioration in moderate to severe $\mathrm{AD}$, for which other treatments are not available. Recent studies carried out in transgenic mice show that memantine decreases the levels of amyloid peptide and prevents synaptic dysfunction. ${ }^{17}$ The activity of protein phosphatase (PP)-2A is compromised in the $\mathrm{AD}$ brain and is believed to be a cause of the abnormal hyperphosphorylation of tau and consequent neurofibrillary degeneration. In those studies, memantine inhibits and reverses the PP-2A inhibition-induced abnormal hyperphosphorylation and accumulation of tau in organotypic cultures of rat hippocampal slices. Such restorative effects of memantine were not detected either with 5,7-dichlorokynurenic acid or with D(-)-2-amino-5-phosphopentanoic acid, N-methyl-Daspartate receptor antagonists, which are active at glycine and glutamate binding sites, respectively. The conclusions of these studies were that memantine inhibits and reverses PP-2A inhibition-induced abnormal hyperphosphorylation of tau/neurofibrillary degeneration, and that this drug might be useful for the treatment of $\mathrm{AD}$ and related tauopathies. ${ }^{19}$

Tau and $\beta$-amyloid peptides are the main contributors to the pathology of AD. Tau localizes via a 3'-UTR region in its mRNA with an axonic signal sequence. ${ }^{18,19}$ Because tau is overexpressed, a disturbance in axonal transport could cause hyperphosphorylation events, leading kinesin not to carry out antegrade transport properly, ${ }^{20,21}$ thus causing a disruption of molecular transport to the axon. Memantine and galantamine produce a reduction in amyloid aggregation, ${ }^{15,17}$ but show no apparent effect on paired helical filament formation.

Although these drugs increase the availability of the neurotransmitter at the synaptic cleft, the neuronal membrane is damaged so, at best, they may slow progression of symptoms for a few months. This highlights a need to repair the neuronal membranes in order to rehabilitate degenerating neurons to respond better to drug treatments.

\section{Neuronal restoration in AD patients}

Why do the drugs available to treat AD have limited efficacy? The answer might be simple. Most of these drugs are 
acetylcholinesterase inhibitors and allow the neurotransmitter, acetylcholine, to remain for longer in the synaptic cleft. The problem we envisage is that degenerative neurons may not have their cholinergic receptors properly positioned on the damaged membrane, and there may even be some receptors not reaching the postsynaptic neuron during trafficking to the dendrite spine. Restoration of neuronal membranes before starting treatment with an acetylcholinesterase inhibitor might enhance the effects of the drugs to produce a prolonged improvement in symptomatology.

\section{Omega-3 acids}

Omega-3 acids have a role in inhibition of ischemic damage $\mathrm{e}^{22}$ and also in increased neurite development, demonstrated when hippocampal neurons are treated with docosahexaenoic acid. Omega-3 acids are also involved in the remodeling of membrane rafts ${ }^{23}$ and in neurogenesis. ${ }^{24}$ Rafts are microdomains of cell membranes, are rich in cholesterol, and are associated with the stabilization of membrane receptors. Membrane rafts have also been shown to be involved in stabilizing the synapse, the $\alpha$-amino-3-hydroxy-5-methyl4-isoxazolepropionic acid ${ }^{25}$ and N-methyl-D-aspartate receptors ${ }^{26}$ and appear to maintain dendritic spines. Rafts are also required for the proper clustering of acetylcholine receptors. ${ }^{27}$ When cell membranes are damaged by reactive oxygen species, as occurs in $\mathrm{AD}$, there is a redistribution of lipids, such as cholesterol and sphingomyelin, because of an increase in membrane fluidity, ${ }^{28}$ and this can lead to changes in the positioning of membrane receptors. Importantly, omega-3 acids have shown the ability to inhibit tau hyperphosphorylation, ${ }^{29}$ which would allow axonal transport restoration. It has been shown that omega- 3 is also capable of reducing amyloid plaque formation..$^{29,30}$

After neuronal membranes are restored, it would be necessary to re-establish the connections between neurons. This process could be aided by drugs like fluoxetine ${ }^{31}$ and escitalopram, ${ }^{32}$ which promote serotonin reuptake. It has been suggested that good recapture of serotonin has a role in the formation of new synapses and neuronal reconnections.

\section{Folic acid}

Folic acid has an important role in neuroplasticity and in the maintenance of neuronal integrity. Folate is a cofactor in one-carbon metabolism, during which it promotes the regeneration of methionine from homocysteine, a highly reactive sulfur-containing amino acid. Methionine can then be converted to S-adenosylmethionine, the principal methyl donor in most biosynthetic methylation reactions. At the cellular level, folate deficiency and hyperhomocysteinemia exert multiple detrimental effects. These include induction of DNA damage, misincorporation of uracil into DNA and altered patterns of DNA methylation. Low folate and elevated homocysteine levels increase the generation of reactive oxygen species, and contribute to excitotoxicity and mitochondrial dysfunction, which might lead to apoptosis. Strong epidemiological and experimental evidence links derangements of one-carbon metabolism to vascular, neurodegenerative, and neuropsychiatric disease, in particular cerebral ischemia, Alzheimer's dementia, and depression. ${ }^{33}$ Therefore, we believe it would be useful to incorporate treatment with folic acid into neuronal rehabilitation therapy.

\section{Ginkgo biloba}

Ginkgo bilboa, a plant extract, has had controversial results in AD patients. ${ }^{34}$ However, some studies have shown a neuroprotective effect (attributed to its high flavonoid content) ${ }^{35}$ and an antioxidant action. ${ }^{36}$ Although it has been suggested that the use of Ginkgo biloba has no benefit in AD patients, there exists some evidence for a beneficial effect from EGb 761 extract. Ginkgo biloba leaves contain a unique kind of flavonoid, and are harvested in South Korea, Japan, and France. ${ }^{37}$

Studies have demonstrated that $\beta$-amyloid peptide treatment induces free radical production and increased glucose uptake, apoptosis, and cell death in PC12 nerve cells. Addition of a standardized extract of Ginkgo biloba leaves, ie, EGb 761, together with $\beta$-amyloid peptide prevented $\beta$-amyloid peptide-induced free radical production, increased glucose uptake, apoptosis, and cell death, in a dose-dependent manner, However, pretreatment of these nerve cells with EGb 761 did not prevent $\beta$-amyloid peptide-induced toxicity, although it did prevent $\beta$-amyloid peptide-induced reactive oxygen species generation. Moreover, the terpene and flavonoid-free EGb 761 extract, HE 208, although able to inhibit a $\beta$-amyloid peptide-induced increase in glucose uptake, failed to protect the cells against $\beta$-amyloid peptideinduced apoptosis and cytotoxicity. These results indicate that the terpenoid and flavonoid constituents of EGb 761, probably acting in combination with other components present in HE 208, are responsible for rescuing neuronal cells from $\beta$-amyloid peptide-induced apoptosis and cell death, their mechanism of action being distinct from their antioxidant properties.

Because pre- and post-treatment with EGb 761 does not protect cell-induced neurotoxicity, the possibility that EGb 761 interacts directly with $\beta$-amyloid peptide 
was investigated. Indeed, in vitro reconstitution studies demonstrated that EGb 761 inhibits, in a dose-dependent manner, the formation of $\beta$-amyloid peptide-derived diffusible neurotoxic soluble ligands, which are thought to be involved in the pathogenesis of AD. ${ }^{38}$ Importantly, Ginkgo biloba acts as a protective agent against the formation of amyloid fibrils, and this effect involves the MAP kinase cascade, SIRT1, and beta NFK. ${ }^{39}$

\section{Resveratrol}

Phytophenols can be arbitrarily divided into the single-ring phenolic acids, the bisphenols, which include stilbenes, tricyclic phenols (flavonoids), and their subclasses, and the oligomeric and polymeric species, proanthocyanidins and anthocyanidins. Phytophenol precursors and stilbenes, including resveratrol and its analogs and conjugates, appear to have a preventive and possibly therapeutic role in atherosclerosis and certain neoplastic and inflammatory conditions. These molecules probably act as free radical scavengers, and might selectively interfere with multiple factors affecting cell division in rapidly and abnormally proliferative mammalian cells. There have been a number of studies published on the natural occurrence, extraction methods, bioavailability, analytical detection, and metabolism of resveratrol, as well as its effects on cancer, the inflammation process, atherosclerosis, and neurons.

Grape extracts are a convenient source of salutary phytochemicals to supplement currently available occidental food. Resveratrol could be added in biosignificant amounts to these extracts if extraction, content, and quality control issues are instituted. ${ }^{40}$

Resveratrol and catechin have been shown to be protective against $\beta$-amyloid peptide in PC12 cells. Many environmental factors, including antioxidants, metal ions, and proteoglycans can modify $\beta$-amyloid peptide (1-41) toxicity in PC12 cells. Protection against reactive oxygen species toxicity is concentration-dependent for both resveratrol and catechin. This protective effect appears to be merely additive, rather than synergistic, and is unlikely to be due to antioxidant activity alone. The difference in chemical and biological activity shown by these compounds for several cell types, as well as the complex toxicity of $\beta$-amyloid peptide (1-41), may explain this synergistic protective effect. Utilization of different compounds with synergistic activity may improve the protective effect against complex toxicity mechanisms. ${ }^{41}$ A polyphenol-rich diet could help to maintain brain homeostasis, prevent oxidation, and keep neurons healthy. ${ }^{42}$

\section{Nimesulide}

Inflammatory processes in the brain are at the basis of the pathogenesis of $\mathrm{AD}$, so nonsteroidal anti-inflammatory drugs have a protective effect in affected individuals. In a study in which quisqualic acid was injected into the right nucleus basalis of rats, excitotoxin induced cholinergic degeneration, an intense glial reaction, and production of inflammatory mediators. Seven days of treatment with intramuscular nimesulide $10 \mathrm{mg} / \mathrm{kg}$ /day strongly attenuated the microglial reaction, reduced the number of inducible nitric oxide synthase-positive cells, and completely abolished increased prostaglandin $\mathrm{E}_{2}$ formation. These data indicate that cyclo-oxygenase-2 (COX-2) inhibitors have potential efficacy in the treatment of AD. ${ }^{43}$

COX-2 is involved in the inflammatory component of the ischemic cascade, playing an important role in delaying progression of brain damage. In one study, the neuroprotective effect of nimesulide was still evident 30 days after an ischemic episode, providing the first experimental evidence that COX-2 inhibitors confer long-lasting neuroprotection. Oral administration of nimesulide was also able to reduce the extent of brain damage significantly, which suggests that its protective effects are independent of the route of administration. This study confirms the ability of COX-2 inhibitors to reduce brain damage induced by cerebral ischemia, and indicates nimesulide can provide neuroprotection when administered for up to 24 hours after an ischemic event. ${ }^{44}$

Nimesulide has been withdrawn from the market in a number of countries because of its side effects, in particular the risk of hepatotoxicity at therapeutic doses. No Englishspeaking country has approved its use, so there are limited data from Phase IV studies. There have been two reports of death in children as a result of Reye's syndrome, a condition commonly associated with aspirin consumption in children under 12 years of age, and which causes numerous detrimental effects, especially to the brain and liver. In these cases, Reye's syndrome was supposedly caused by consumption of nimesulide. In addition, nimesulide was observed to have a dangerously rapid antipyretic effect in children, and is not recommended for reduction of fever under $40^{\circ} \mathrm{C}$ (approximately $101^{\circ} \mathrm{F}$ ). However, physicians often succumb to parental pressure to lower their child's fever. In countries like Finland, Spain, and Turkey, use of nimesulide has been prohibited in both children and adults, because of its hepatotoxicity, excessive antipyretic effect, and the potential to cause Reye's syndrome. The European Drug Evaluation Agency is currently evaluating the status of nimesulide for an all-European approach. 
Despite the adverse effects associated with nimesulide, it is beneficial if only given for a limited period of time and, like other nonsteroidal anti-inflammatory drugs, should not be used in children.

\section{Recommendations}

To date, we have followed two AD patients trying this therapy. Both patients were on drug treatment but showing no improvement. In addition to their existing treatment, they started our proposed therapy approximately one year ago, with satisfactory results so far.

\section{Case I}

An 83-year-old woman was diagnosed with AD about 10 years ago. At the time the proposed therapy was initiated, the patient presented with confusion about money, words, and the placing of objects, in addition to difficulty in remembering certain activities and even recognizing her relatives. She had been taking rivastigmine, but after two years she started showing marked cognitive decline. After one year taking our proposed therapy, she now shows an improvement in memory, fluidity of language, and recognizes her relatives again. One of her sons said that the disease seems to have stopped, and that her brain appears to be regaining functionality.

\section{Case 2}

A man with a dramatic picture of $\mathrm{AD}$ had presented with very rapid cognitive decline, despite being treated with memantine. His wife made the decision to start this therapy, and his memory and conversational coherence has improved markedly. The only residual sign of the disease in this man is occasional mild aggression.

\section{Discussion}

Omega-3 acid could help restoration of neuronal membranes to the $\mathrm{AD}$ brain, allowing proper positioning of postsynaptic receptors, and enabling existing drug treatment to be more effective. Tau dephosphorylation would restore axonal transport of synaptic vesicles, and thus improve neurotransmitter release at the axon terminals. Once the membranes have been repaired, it would be important to induce the formation and maintenance of new synapses (Figure 1) by fluoxetine

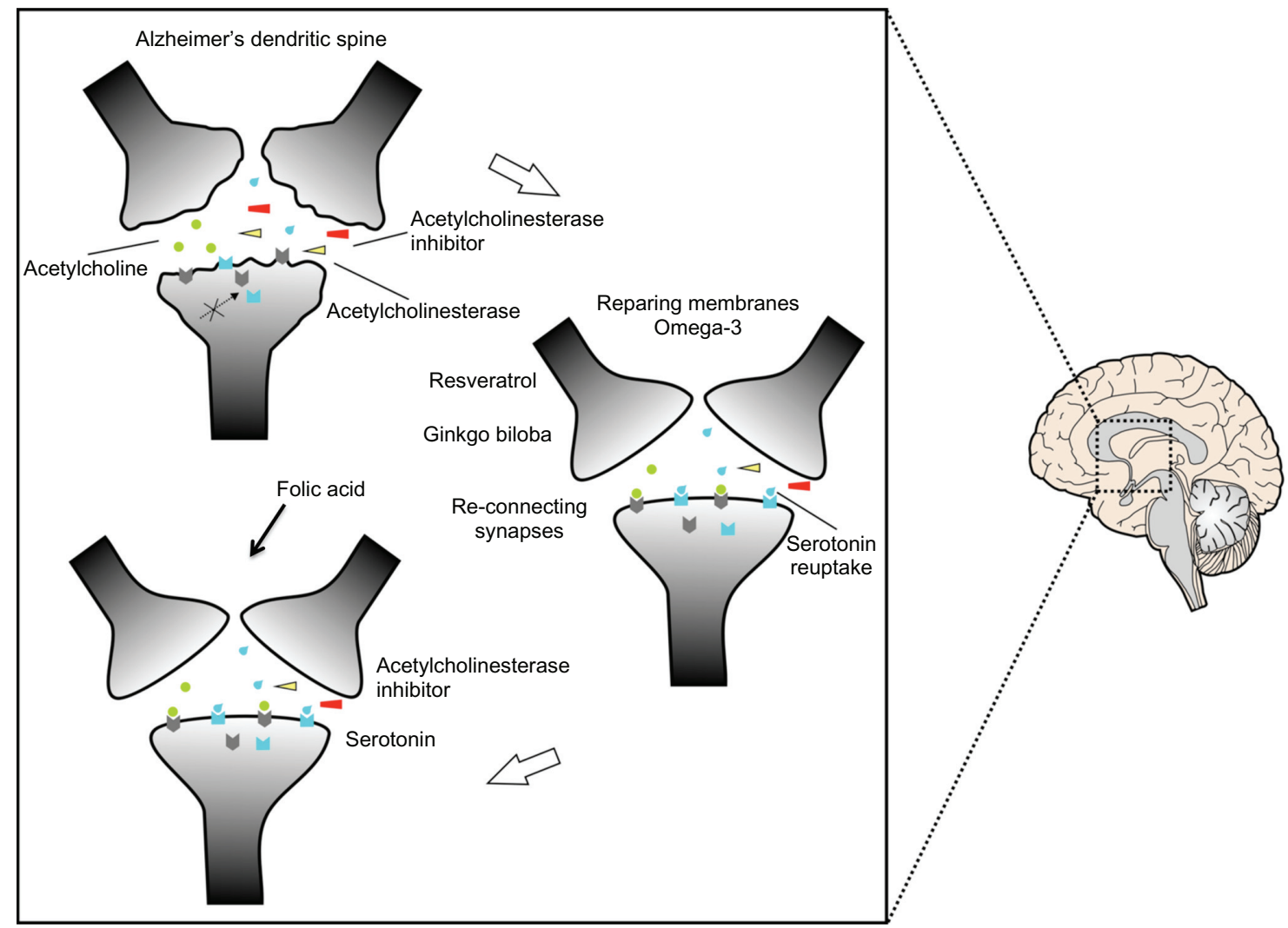

Figure I Restoration of the dendritic spine. Dendrites damaged as a result of Alzheimer's disease would result in abnormal neurotransmission because the receptors are not located in the right site as a result of the effect of neurodegeneration. The repair process is shown, beginning with omega- 3 to allow repair of membranes and thus locate the receptors in the right places for effective neurotransmission. Serotonergic neurons would help in the formation of circuits due to the use of serotonin reuptake. Resveratrol and Ginkgo biloba serve as antioxidants and in the process of memory, respectively. Folic acid maintains the integrity of the newly repaired circuits. 


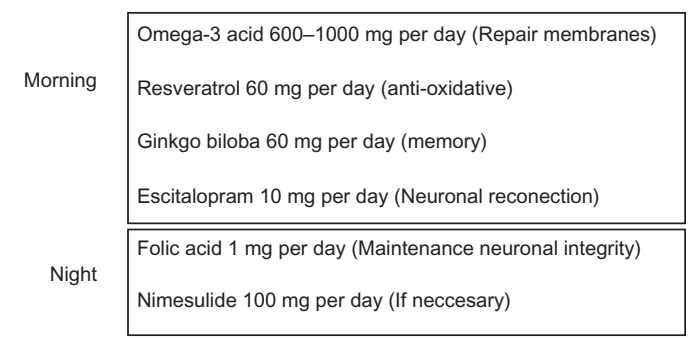

Drugs for Alzheimer's disease (memantine, galantamine, as Medical Doctor indicated)

Figure 2 Alternative therapy to rehabilitate a neuron.

or escitalopram and folic acid. Serotonin regulates neuronal morphology and the reconnection between neurons ${ }^{45}$ its pathway interacts with that of acetylcholine, ${ }^{46}$ and it also has a role in memory impairment. ${ }^{47,48}$ A powerful antioxidant, such as resveratrol, and the activation of memory processes induced by Ginkgo bilboa, would improve the effects of membrane repair and formation of synapses. All of this together might then rehabilitate the $\mathrm{AD}$ brain to enable institution of drug treatment producing a better outcome (Figure 2). Ongoing neurodegenerative processes and the mechanisms of action of the drugs available have failed to cure $\mathrm{AD}^{49}$ because they are brain enhancers. ${ }^{50}$

We believe this rehabilitating therapy in combination with drug treatment gives us a good possibility for improving the quality of life of both $\mathrm{AD}$ patients and their relatives, who also suffer the consequences of the disease. We propose an experimental strategy to evaluate the effects of this therapy (Figure 3). Of course, this should be tested in more AD patients in the near future.
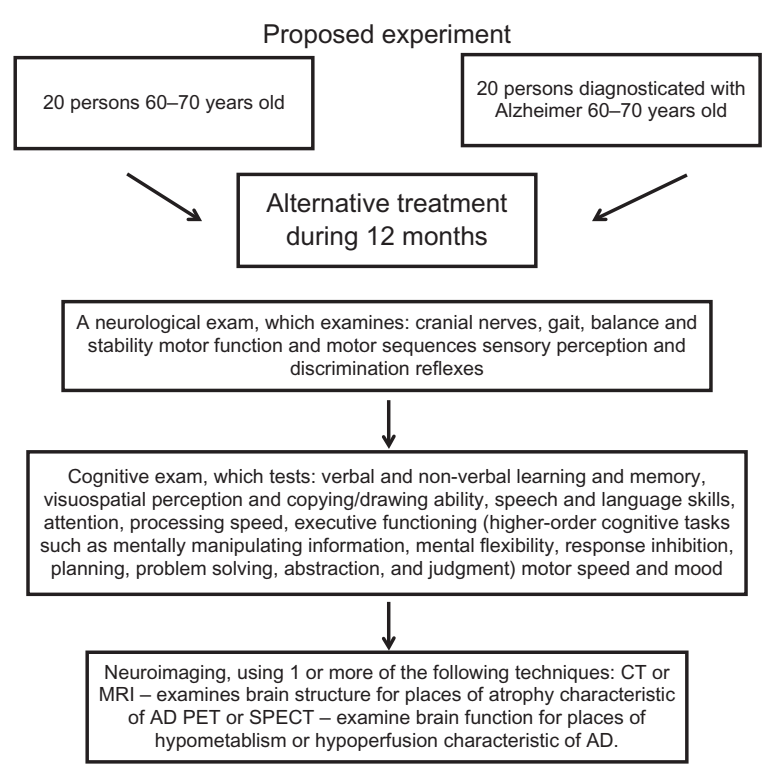

Figure 3 Proposed experiment to probe the alternative therapy.
We must bear in mind that $\mathrm{AD}$ has a slow progression and the neuronal damage occurs over a long period of time, to such an extent that it becomes impossible to prevent brain cell degeneration. At the last congress of The Society of Neuroscience at Chicago in 2009, the introduction of natural products to treat $\mathrm{AD}$ was recommended.

The alternative therapy we propose here could take over a year to have an effect, but it might be really helpful in patients with mild to moderate AD. However, in the advanced stages of the disease, this therapy would not be appropriate due to the large amount of neuronal loss. Although this therapy requires patience and cooperation from both patients and their relatives, we believe it provides a good option to improve treatment outcomes in AD.

\section{Disclosure}

The authors report no conflicts of interest in this work.

\section{References}

1. Perez-Tur J. Genetics and Alzheimer's disease. Rev Neurol. 2000;30: 161-169.

2. Lopez de Munain A. Classification of mitochondrial diseases. Rev Neurol. 1998;26 Supp1 1:S9-S14.

3. Hardy J, Allsop D. Amyloid deposition as the central event in the aetiology of Alzheimer's disease. Trends Pharmacol Sci. 1991;12: 383-388.

4. Jarrett JT, Lansbury PTJ. Seeding “one-dimensional crystallization” of amyloid: A pathogenic mechanism in Alzheimer's disease and scrapie? Cell. 1993;73:1055-1058

5. Hu X, Hicks CW, He W, et al. BACE1 modulates myelination in the central and peripheral nervous system. Nat Neurosci. 2006;9: 1520-1525.

6. Willem M, Lammich S, Haass C. Function, regulation and therapeutic properties of beta-secretase (BACE1). Semin Cell Dev Biol. 2009;20: $175-182$.

7. Nguyen V, Hawkins C, Bergeron C, et al. Loss of nicastrin elicits an apoptotic phenotype in mouse embryos. Brain Res. 2006;1086:76-84.

8. Gauthier S, Scheltens P. Can we do better in developing new drugs for Alzheimer's disease? Alzheimers Dement. 2009;5:489-491.

9. Qizilbash N, Birks J, Lopez Arrieta J, Lewington S, Szeto S. WITHDRAWN: Tacrine for Alzheimer's disease. Cochrane Database Syst Rev. 2007:CD000202.

10. Alfirevic A, Mills T, Carr D, et al. Tacrine-induced liver damage: An analysis of 19 candidate genes. Pharmacogenet Genomics. 2007;17: 1091-1100.

11. Tsuno N. Donepezil in the treatment of patients with Alzheimer's disease. Expert Rev Neurother. 2009;9:591-598.

12. Winblad B. Donepezil in severe Alzheimer's disease. Am J Alzheimers Dis Other Demen. 2009;24:185-192.

13. Birks J, Grimley Evans J, Iakovidou V, Tsolaki M, Holt FE. Rivastigmine for Alzheimer's disease. Cochrane Database Syst Rev. 2009:CD001191.

14. Annicchiarico R, Federici A, Pettenati C, Caltagirone C. Rivastigmine in Alzheimer's disease: Cognitive function and quality of life. Ther Clin Risk Manag. 2007;3:1113-1123.

15. Matharu B, Gibson G, Parsons R, et al. Galantamine inhibits betaamyloid aggregation and cytotoxicity. J Neurol Sci. 2009;280:49-58.

16. Melo JB, Sousa C, Garcao P, Oliveira CR, Agostinho P. Galantamine protects against oxidative stress induced by amyloid-beta peptide in cortical neurons. Eur J Neurosci. 2009;29:455-464. 
17. Martinez-Coria H, Green KN, Billings LM, et al. Memantine improves cognition and reduces Alzheimer's-like neuropathology in transgenic mice. Am J Pathol. 2010;176:870-880.

18. Aranda-Abreu GE, Behar L, Chung S, Furneaux H, Ginzburg I. Embryonic lethal abnormal vision-like RNA-binding proteins regulate neurite outgrowth and tau expression in PC12 cells. J Neurosci. 1999;19:6907-6917.

19. Aronov S, Aranda G, Behar L, Ginzburg I. Axonal tau mRNA localization coincides with tau protein in living neuronal cells and depends on axonal targeting signal. J Neurosci. 2001;21:6577-6587.

20. Dubey M, Chaudhury P, Kabiru H, Shea TB. Tau inhibits anterograde axonal transport and perturbs stability in growing axonal neurites in part by displacing kinesin cargo: Neurofilaments attenuate tau-mediated neurite instability. Cell Motil Cytoskeleton. 2008;65:89-99.

21. Lippens G, Sillen A, Landrieu I, et al. Tau aggregation in Alzheimer's disease: What role for phosphorylation? Prion. 2007;1:21-25.

22. Relton JK, Strijbos PJ, Cooper AL, Rothwell NJ. Dietary N-3 fatty acids inhibit ischaemic and excitotoxic brain damage in the rat. Brain Res Bull. 1993;32:223-226.

23. Fan YY, McMurray DN, Ly LH, Chapkin RS. Dietary (n-3) polyunsaturated fatty acids remodel mouse T-cell lipid rafts. J Nutr. 2003;133: 1913-1920.

24. Beltz BS, Tlusty MF, Benton JL, Sandeman DC. Omega-3 fatty acids upregulate adult neurogenesis. Neurosci Lett. 2007;415:154-158.

25. Hering H, Lin CC, Sheng M. Lipid rafts in the maintenance of synapses, dendritic spines, and surface AMPA receptor stability. J Neurosci. 2003;23:3262-3271.

26. Hou Q, Huang Y, Amato S, Snyder SH, Huganir RL, Man HY. Regulation of AMPA receptor localization in lipid rafts. Mol Cell Neurosci. 2008;38:213-223.

27. Stetzkowski-Marden F, Recouvreur M, Camus G, Cartaud A, Marchand S, Cartaud J. Rafts are required for acetylcholine receptor clustering. J Mol Neurosci. 2006;30:37-38.

28. Clement AB, Gimpl G, Behl C. Oxidative stress resistance in hippocampal cells is associated with altered membrane fluidity and enhanced nonamyloidogenic cleavage of endogenous amyloid precursor protein. Free Radic Biol Med. 2010;48:1236-1241.

29. Ma QL, Yang F, Rosario ER, et al. Beta-amyloid oligomers induce phosphorylation of tau and inactivation of insulin receptor substrate via c-Jun N-terminal kinase signaling: suppression by omega-3 fatty acids and curcumin. J Neurosci. 2009;29:9078-9089.

30. Amtul Z, Uhrig M, Rozmahel RF, Beyreuther K. Structural basis for the differential effects of omega-3 and omega- 6 fatty acids on Abeta production and amyloid plaques. J Biol Chem. 2010 Oct 22. [Epub ahead of print].

31. Wang JW, David DJ, Monckton JE, Battaglia F, Hen R. Chronic fluoxetine stimulates maturation and synaptic plasticity of adult-born hippocampal granule cells. J Neurosci. 2008;28:1374-1384.

32. Lucas G, Du J, Romeas T, et al. Selective serotonin reuptake inhibitors potentiate the rapid antidepressant-like effects of serotonin4 receptor agonists in the rat. PLoS One. 2010;5:e9253.

33. Kronenberg G, Colla M, Endres M. Folic acid, neurodegenerative and neuropsychiatric disease. Curr Mol Med. 2009;9:315-323.

34. Oken BS, Storzbach DM, Kaye JA. The efficacy of Ginkgo biloba on cognitive function in Alzheimer's disease. Arch Neurol. 1998;55: 1409-1415.
35. Bastianetto S, Zheng WH, Quirion R. The Ginkgo biloba extract (EGb 761) protects and rescues hippocampal cells against nitric oxide-induced toxicity: Involvement of its flavonoid constituents and protein kinase C. J Neurochem. 2000;74:2268-2277.

36. Grundman M, Grundman M, Delaney P. Antioxidant strategies for Alzheimer's disease. Proc Nutr Soc. 2002;61:191-202.

37. Drieu K. Preparation and definition of Ginkgo biloba extract. Presse Med. 1986;15:1455-1457.

38. Yao Z, Drieu K, Papadopoulos V. The Ginkgo biloba extract EGb 761 rescues the PC12 neuronal cells from beta-amyloid-induced cell death by inhibiting the formation of beta-amyloid-derived diffusible neurotoxic ligands. Brain Res. 2001;889:181-190.

39. Longpre F, Garneau P, Christen Y, Ramassamy C. Protection by EGb 761 against beta-amyloid-induced neurotoxicity: Involvement of NF-kappaB, SIRT1, and MAPKs pathways and inhibition of amyloid fibril formation. Free Radic Biol Med. 2006;41:1781-1794

40. Sovak M. Grape extract, resveratrol, and its analogs: A review. J Med Food. 2001;4:93-105.

41. Conte A, Pellegrini S, Tagliazucchi D. Synergistic protection of PC12 cells from beta-amyloid toxicity by resveratrol and catechin. Brain Res Bull. 2003;62:29-38.

42. Rossi L, Mazzitelli S, Arciello M, Capo CR, Rotilio G. Benefits from dietary polyphenols for brain aging and Alzheimer's disease. Neurochem Res. 2008;33:2390-2400.

43. Scali C, Prosperi C, Vannucchi MG, Pepeu G, Casamenti F. Brain inflammatory reaction in an animal model of neuronal degeneration and its modulation by an anti-inflammatory drug: Implication in Alzheimer's disease. Eur J Neurosci. 2000;12:1900-1912.

44. Candelario-Jalil E, Alvarez D, Gonzalez-Falcon A, et al. Neuroprotective efficacy of nimesulide against hippocampal neuronal damage following transient forebrain ischemia. Eur J Pharmacol. 2002;453:189-195.

45. Daubert EA, Condron BG. Serotonin: A regulator of neuronal morphology and circuitry. Trends Neurosci. 2010;33:424-434.

46. Altman HJ, Stone WS, Ogren SO. Evidence for a possible functional interaction between serotonergic and cholinergic mechanisms in memory retrieval. Behav Neural Biol. 1987;48:49-62.

47. Buhot MC, Martin S, Segu L. Role of serotonin in memory impairment. Ann Med. 2000;32:210-221.

48. Dougherty JJ, Nichols RA. Cross-regulation between colocalized nicotinic acetylcholine and 5-HT3 serotonin receptors on presynaptic nerve terminals. Acta Pharmacol Sin. 2009;30:788-794.

49. National Institutes of Health. Alzheimer's Disease Medications. 2008 Available from: http:/www.nia.nih.gov/Alzheimers/Publications/ medicationsfs.htm. Accessed on January 18, 2010.

50. Stix G. Turbocharging the brain. Sci Am. 2009;301:46-49.

51. Jha AM, Abha. Assessment of cytotoxic and clastogenic effects of nimesulide: An NSAID drug in somatic cells of BALB/c mice in vivo. Drug Chem Toxicol. 2010;33:204-208.

52. Goncalves MB, Williams EJ, Yip P, Yanez-Munoz RJ, Williams G, Doherty P. The COX-2 inhibitors, meloxicam and nimesulide, suppress neurogenesis in the adult mouse brain. Br J Pharmacol. 2010;159: $1118-1125$.
Clinical Interventions in Aging

\section{Publish your work in this journal}

Clinical Interventions in Aging is an international, peer-reviewed journal focusing on evidence-based reports on the value or lack thereof of treatments intended to prevent or delay the onset of maladaptive correlates of aging in human beings. This journal is indexed on PubMed Central, MedLine, the American Chemical Society's 'Chemical

\section{Dovepress}

Abstracts Service' (CAS), Scopus and the Elsevier Bibliographic databases. The manuscript management system is completely online and includes a very quick and fair peer-review system, which is all easy to use. Visit http://www.dovepress.com/testimonials.php to read real quotes from published authors. 